Pacific Journal of Mathematic 


\section{ORDER SUMS OF DISTRIBUTIVE LATTICES}

\section{RAYMoND BALbes AND AlFREd HoRN}

In this paper, the authors define the order sum of a family of distributive lattices which is indexed by a partially ordered set $P$. The order sum reduces to the free product when $P$ is trivially ordered, and to the ordinal sum when $P$ is simply ordered.

It is proved that the order sum of conditionally implicative lattices is conditionally implicative, and that every projective distributive lattice is conditionally implicative. The second half of the paper investigates conditions under which the order sum of projective lattices is projective. It is shown that if $\left\{L_{\alpha} \mid \alpha \in P\right\}$ is a family of distributive lattices having largest and smallest elements, then the order sum of the family is projective if and only if each $L_{\alpha}$ is projective, and $P$ is such that the order sum of the family $\left\{M_{\alpha} \mid \alpha \in P\right\}$ of one-element lattices $M_{\alpha}$ is projective.

\section{Existence and properties of order sums.}

Definition 1.1. Let $\left\{L_{\alpha} \mid \alpha \in P\right\}$ be a family of distributive lattices whose index set $P$ is partially ordered. A distributive lattice $L$ is called the order sum of the family if for each $\alpha \in P$ there exists a monomorphism $\varphi_{\alpha}: L_{\alpha} \rightarrow L$ such that:

(1.1) $L$ is generated by $\bigcup_{\alpha \in P} \varphi_{\alpha}\left(L_{\alpha}\right)$.

(1.2) If $\alpha<\beta$ and $x \in L_{\alpha}, y \in L_{\beta}$, then $\varphi_{\alpha}(x)<\varphi_{\beta}(y)$.

(1.3) If $M$ is any distributive lattice and $\left\{f_{\alpha}: L_{\alpha} \rightarrow M \mid \alpha \in P\right\}$ is a family of homomorphisms such that whenever $\alpha<\beta, f_{\alpha}(x) \leqq f_{\beta}(y)$ for all $x \in L_{\alpha}$ and $y \in L_{\beta}$, then there exists a homomorphism $f: L \rightarrow M$ such that $f \varphi_{\alpha}=f_{\alpha}$ for all $\alpha \in P$.

The existence of the order sum will be proved in Theorem 1.7.

THEOREM 1.2. If $L$ and $M$ are order sums of $\left\{L_{\alpha} \mid \alpha \in P\right\}$, then $L \cong M$.

Proof. Let $\varphi_{\alpha}: L_{\alpha} \rightarrow L$ and $\psi_{\alpha}: L_{\alpha} \rightarrow M$ be monomorphisms as in Definition 1.1. By (1.3), there exist homomorphisms $f: M \rightarrow L$ and $g: L \rightarrow M$ such that $f \psi_{\alpha}=\varphi_{\alpha}$ and $g \varphi_{\alpha}=\psi_{\alpha}$. We have $f g \varphi_{\alpha}=f \psi_{\alpha}=\phi_{\alpha}$ for each $\alpha$, and therefore by (1.1), $f g=I_{L}$, the identity function on $L$. Similarly $g f=I_{M}$. Thus $g=f^{-1}$ and $f$ is an isomorphism.

By Theorem 1.2, the order sum of a family $\left\{L_{\alpha} \mid \alpha \in P\right\}$ is unique up to isomorphism. We denote the order sum by $\Sigma_{\alpha \in P} L_{\alpha}$. If $P$ is 
trivially ordered, in the sense that $\alpha \leqq \beta$ only when $\alpha=\beta$, then the order sum reduces by very definition to the free product [2].

Definition 1.3. Let $\left\{L_{\alpha} \mid \alpha \in P\right\}$ be a family of pairwise disjoint lattices indexed by a chain $P$. The ordinal sum $L$ of the family is the set $\bigcup_{\alpha \in P} L_{\alpha}$ with the following partial order: if $x \in L_{\alpha}$ and $y \in L_{\beta}$, then $x \leqq y$ if and only if either $\alpha<\beta$, or $\alpha=\beta$ and $x \leqq y$ in the original order of $L_{\alpha}$. If the lattices $L_{\alpha}$ are not pairwise disjoint, then the ordinal sum of the $L_{\alpha}$ is defined to be the ordinal sum of pairwise disjoint lattices $\widetilde{L}_{\alpha}$ such that $\widetilde{L}_{\alpha} \cong L_{\alpha}$. If $P$ is the chain $\{0,1\}$ with $0<1$, then the ordinal sum of $\left\{L_{0}, L_{1}\right\}$ is denoted by $L_{0} \oplus L_{1}$.

THEOREM 1.4. If $P$ is a chain, then the ordinal sum $L$ of $\left\{L_{\alpha} \mid \alpha \in P\right\}$ is the order sum $\Sigma_{\alpha \in P} L_{\alpha}$.

Proof. We may assume the $L_{\alpha}$ are pairwise disjoint. Let $\varphi_{\alpha}: L_{\alpha} \rightarrow L$ be the inclusion map. Obviously conditions (1.1) and (1.2) hold. Suppose the $f_{\alpha}$ are as in (1.3). Let $f: L \rightarrow M$ be the union (combined function) of the $f_{\alpha}$. It is clear that $f$ is a homomorphism and $f \varphi_{\alpha}=f_{\alpha}$.

Corollary 1.5. If $P$ is a chain, and for each $\alpha \in P, L_{\alpha}$ is a one-element lattice, then $\Sigma_{\alpha \in P} L_{\alpha} \cong P$.

Proof. In this case the ordinal sum of the $L_{\alpha}$ is obviously isomorphic with $P$.

THEOREM 1.6. Let $\left\{x_{i} \mid i \in I\right\}$ be a family of distinct variables. Let $E$ be a set of inequalities of the form $x_{i_{1}} \cdots \cdot x_{i_{m}} \leqq x_{j_{1}}+\cdots+x_{j_{n}}$, where the indices are in $I$. Then there exists a distributive lattice $L$ which is generated by a family $\left\{a_{i} \mid i \in I\right\}$ of elements such that

(1.4) $\left\{a_{i} \mid i \in I\right\}$ satisfies every inequality in $E$.

(1.5) If $M$ is a distributive lattice which contains a family $\left\{b_{i} \mid i \in I\right\}$ which satisfies every inequality in $E$, then there exists a homomorphism $f: L \rightarrow M$ such that $f\left(a_{i}\right)=b_{i}$ for all $i \in I$.

Proof. This is a special case of a general theorem of algebra, since the class of distributive lattices is equational. A different proof for the case of distributive lattices is given in [1, Th. 1.9].

Theorem 1.7. If $\left\{L_{\alpha} \mid \alpha \in P\right\}$ is a family of distributive lattices, and $P$ is a partially ordered set, then $\sum_{\alpha \in P} L_{\alpha}$ exists. 
Proof. Let $\left\{x_{\alpha, i} \mid \alpha \in P, i \in L_{\alpha}\right\}$ be a family of distinct variables. Let $E_{\alpha}$ be the set of all inequalities $x_{\alpha, i_{1}}, \cdots \cdot x_{\alpha, i_{m}} \leqq x_{\alpha, j_{1}}+\cdots+x_{\alpha, j_{n}}$ such that $i_{1} \cdots \cdot i_{m} \leqq j_{1}+\cdots+j_{n}$ in $L_{\alpha}$. Let $E$ be the union of the $E_{\alpha}, \alpha \in P$, together with all inequalities $x_{\alpha, i} \leqq x_{\beta, j}$ such that $\alpha<\beta, i \in L_{\alpha}$ and $j \in L_{\beta}$. By Theorem 1.6, there exists a distributive lattice $L$ generated by a family $\left\{a_{\alpha, i} \mid \alpha \in P, i \in L_{\alpha}\right\}$ satisfying (1.4) and (1.5). We will show $L=\sum_{\alpha \in P} L_{\alpha}$.

Define $\varphi_{\alpha}: L_{\alpha} \rightarrow L$ by $\varphi_{\alpha}(i)=a_{\alpha, i}$. To show $\varphi_{\alpha}$ is a homomorphism, let $i \in L_{\alpha}, j \in L_{\alpha}$, and let $k=i+j$. Since $i \leqq k, j \leqq k$, and $k \leqq i+j$, we have $a_{\alpha, i} \leqq a_{\alpha, k}, a_{\alpha, j} \leqq a_{\alpha, k}$, and $a_{\alpha, k} \leqq a_{\alpha, i}+a_{\alpha, j}$, since $\left\{a_{\alpha, i} \mid i \in L_{\alpha}\right\}$ satisfies $E_{\alpha}$. It follows that $a_{\alpha, k}=a_{\alpha, i}+a_{\alpha, j}$, and so $\varphi_{\alpha}(i+j)=$ $\varphi_{\alpha}(i)+\varphi_{\alpha}(j)$. Similarly $\varphi_{\alpha}(i j)=\varphi_{\alpha}(i) \varphi_{\alpha}(j)$.

To show that $\varphi_{\alpha}$ is injective, suppose $a_{\alpha, i}=a_{\alpha, j}$ but $i \not j$. There exists a prime filter $F$ in $L_{\alpha}$ such that $i \in F$ and $j \notin F$. Let $M=\{0,1\}$ be the chain such that $0<1$. Let $b_{\alpha, k}=1$ if $k \in F, b_{\alpha, k}=0$ if $k \in L_{\alpha}-F, b_{\beta, k}=1$ if $\beta \notin \alpha, k \in L_{\beta}$, and $b_{\beta, k}=0$ if $\beta<\alpha, k \in L_{\beta}$. Since $F$ is a prime filter, $\left\{b_{\alpha, k} \mid k \in L_{\alpha}\right\}$ satisfies all inequalities in $E_{\alpha}$. Clearly if $\beta \neq \alpha,\left\{b_{\beta, k} \mid k \in L_{\beta}\right\}$ satisfies all inequalities in $E_{\beta}$. If $\beta<\gamma$, then $b_{\beta, k} \leqq b_{\gamma, r}$ for all $k \in L_{\beta}, r \in L_{\gamma}$. Indeed if not, $b_{\beta, k}=1$ and $b_{\gamma, r}=0$, which implies $\gamma \leqq \alpha$ and therefore $\beta<\alpha$, contradicting $b_{\beta, k}=1$. Thus $\left\{b_{\beta, k} \mid \beta \in P, k \in L_{\beta}\right\}$ satisfies all inequalities in $E$. By Theorem 1.6, there exists a homomorphism $f: L \rightarrow M$ such that $f\left(a_{\beta, k}\right)=b_{\beta, k}$. In particular $f\left(a_{\alpha, i}\right)=1$ and $f\left(a_{\alpha, j}\right)=0$. This contradicts $a_{\alpha, i}=a_{\alpha, j}$.

We now verify (1.1), (1.2) and (1.3). $L$ is generated by $\bigcup_{\alpha \in P} \varphi_{\alpha}\left(L_{\alpha}\right)$, since $L$ is generated by $\left\{a_{\alpha, i} \mid \alpha \in P, i \in L_{\alpha}\right\}$. Next suppose $\alpha<\beta, i \in L_{\alpha}$, and $j \in L_{\beta}$. Then $a_{\alpha, i} \leqq a_{\beta, j}$ since $x_{\alpha, i} \leqq x_{\beta, j}$ is in $E$. To show $a_{\beta, j} \not \leq a_{\alpha, i}$, let $M=\{0,1\}$ and let $b_{\gamma, k}=1$ if $\gamma \not \alpha, k \in L_{\gamma}$, and $b_{\gamma, k}=0$ if $\gamma \leqq \alpha, k \in L_{\gamma}$. Clearly $\left\{b_{\gamma, k} \mid \gamma \in P, k \in L_{\gamma}\right\}$ satisfies all inequalities in $E$. By Theorem 1.6, there exists a homomorphism $f: L \rightarrow M$ such that $f\left(a_{\beta, j}\right)=1$ and $f\left(a_{\alpha, i}\right)=0$. Therefore $a_{\beta, j} \geqq a_{\alpha, i}$.

Finally suppose we have homomorphisms $f_{\alpha}$ as in (1.3). Let $b_{\alpha, i}=f_{\alpha}(i)$. Obviously $\left\{b_{\alpha, i} \mid \alpha \in P, i \in L_{\alpha}\right\}$ satisfies all inequalities in $E$. By Theorem 1.6, there exists a homomorphism $f: L \rightarrow M$ such that $f\left(a_{\alpha, i}\right)=b_{\alpha, i}$ and therefore $f \varphi_{\alpha}=f_{\alpha}$.

In $[2, \S 9]$ a construction is given for the free product of a family of distributive lattices which implies that the Stone space of the free product of $\left\{L_{\alpha} \mid \alpha \in I\right\}$ is the Cartesian product of the Stone spaces of the $L_{\alpha}$. This statement and the construction are not accurate. The following theorem describes the set of all prime filters of $\sum_{a \in P} L_{\alpha}$ in the general case where $P$ is partially ordered.

THEOREM 1.8. Let $\Im$ be the set of all functions $f$ on $P$ such that

(1.6) for each $\alpha \in P, f(\alpha)$ is either a prime filter in $L_{\alpha}$ or 
$f(\alpha)=\varnothing$, or $f(\alpha)=L_{\alpha}$

(1.7) if $\alpha<\beta$ and $f(\alpha) \neq \varnothing$, then $f(\beta)=L_{\beta}$

(1.8) for some $\alpha, f(\alpha) \neq \varnothing$, and for some $\alpha, f(\alpha) \neq L_{\alpha}$

There is a one-to-one correspondence between $\widetilde{F}$ and the set of all prime filters in $L=\sum_{\alpha \in P} L_{\alpha}$ such that if $F$ is the prime filter corresponding to $f$, then $f(\alpha)=\varphi_{\alpha}^{-1}\left(F \cap \varphi_{\alpha}\left(L_{\alpha}\right)\right)$. Here $\varphi_{\alpha}$ is the monomorphism described in Definition 1.1.

Proof. Let $F$ be a prime filter in $\sum_{\alpha \in_{P}} L_{\alpha}$, and let $f$ be the function on $P$ such that $f(\alpha)=\varphi_{\alpha}^{-1}\left(F \cap \varphi_{\alpha}\left(L_{\alpha}\right)\right)$. We show $f \in \mathfrak{F}$. Since $\varphi_{\alpha}\left(L_{\alpha}\right)$ is a sublattice of $\sum_{\alpha \in P} L_{\alpha},(1.6)$ holds. Conditions (1.7) and (1.8) follow immediately from (1.2) and (1.1) respectively. Conversely, if $f \in \mathfrak{F}$, let $M=\{0,1\}$, and let $f_{\alpha}: L_{\alpha} \rightarrow M$ be such that $f_{\alpha}(x)=1$ if $x \in f(\alpha)$ and $f_{\alpha}(x)=0$ if $x \in L_{\alpha}-f(\alpha)$. Clearly $\left\{f_{\alpha} \mid \alpha \in P\right\}$ satisfies the hypothesis of (1.3). Therefore there exists a homomorphism $g: L \rightarrow M$ such that $g \varphi_{\alpha}=f_{\alpha}$. If we set $F=\{x \in L \mid g(x)=1\}, F$ is a prime filter and $f(\alpha)=\varphi_{\alpha}^{-1}\left(F \cap \varphi_{\alpha}\left(L_{\alpha}\right)\right)$.

The following lemma describes the inequalities which hold within an order sum.

LEMMA 1.9. Suppose $L=\sum_{\alpha \in P} L_{\alpha}$, and $\varphi_{\alpha}$ is the monomorphism of Definition 1.1. Assume $\alpha_{1}, \cdots, \alpha_{m}$ are distinct members of $P, \beta_{1}, \cdots, \beta_{n}$ are distinct members of $P, i_{r} \in L_{\alpha_{r}}$ for $1 \leqq r \leqq m$, and $j_{s} \in L_{\beta s}$ for $1 \leqq s \leqq n$. Then

(1.9) $\varphi_{\alpha_{1}}\left(i_{1}\right) \cdot \cdots \cdot \varphi_{\alpha_{m}}\left(i_{m}\right) \leqq \varphi_{\beta_{1}}\left(j_{1}\right)+\cdots+\varphi_{\beta_{n}}\left(j_{n}\right)$ if and only if for some $r$ and $s$ either $\alpha_{r}<\beta_{s}$ or $\alpha_{r}=\beta_{s}$ and $i_{r} \leqq j_{s}$.

Proof. The condition is obviously sufficient. By changing indices, we may assume $\alpha_{1}=\beta_{1}, \cdots, \alpha_{p}=\beta_{p}$, and $\left\{\alpha_{p+1}, \cdots, \alpha_{m}\right\}$ is disjoint from $\left\{\beta_{p+1}, \cdots, \beta_{n}\right\}$, where $p$ may be 0 . Let $M$ be the chain $\{0,1\}$. Suppose the condition fails to hold. Then there exist prime filters $F_{k}$ in $L_{\alpha_{k}}, 1 \leqq k \leqq p$, such that $i_{k} \in F_{k}$ and $j_{k} \notin F_{k}$. Let $f$ be the function on $P$ such that

$$
\begin{aligned}
f\left(\alpha_{k}\right) & =F_{k}, & & 1 \leqq k \leqq p \\
f\left(\alpha_{k}\right) & =L_{\alpha_{k}}, & & p+1 \leqq k \leqq m \\
f(\alpha) & =L_{\alpha}, & & \text { if } \alpha>\alpha_{k} \text { for some } k, 1 \leqq k \leqq m
\end{aligned}
$$

and

$$
f(\alpha)=\varnothing \quad \text { otherwise. }
$$

By Theorem 1.8, there exists a prime filter $F$ in $L$ such that $\varphi_{\alpha}^{-1}\left(F \cap \varphi_{\alpha}\left(L_{\alpha}\right)\right)=f(\alpha)$. This implies $\varphi_{\alpha_{k}}\left(i_{k}\right) \in F$ for $1 \leqq k \leqq m$, and 
$\varphi_{\beta_{k}}\left(j_{k}\right) \notin F$ for $1 \leqq k \leqq n$. Since $F$ is a prime filter, (1.9) cannot hold.

Definition 1.10. If $P$ is a partially ordered set, we denote by $P^{*}$ the order sum $\sum_{\alpha \in P} M_{\alpha}$, where for each $\alpha, M_{\alpha}$ is a one-element lattice. By Lemma 1.9, the set $\bigcup_{\alpha \in P} \varphi_{\alpha}\left(M_{\alpha}\right)$ is isomorphic with $P$. Therefore we will identify the lone member of $\varphi_{\alpha}\left(M_{\alpha}\right)$ with $\alpha$, and $P$ will be a generating subset of $P^{*}$. If $P$ is a lattice, $P$ will not usually be a sublattice of $P^{*} . \quad P^{*}$ is characterized by the property that every order preserving function on $P$ to a distributive lattice $M$ can be extended uniquely to a homomorphism on $P^{*}$ to $M$.

THEOREM 1.11. If $Q$ is any nonempty subset of $P$, then $Q^{*}$ is isomorphic with the sublattice $N$ of $P^{*}$ which is generated by $Q$.

Proof. Let $f$ be an order preserving function on $Q$ to a distributive lattice $M$. By [1, Lemma 1.7.] $f$ can be extended to a homomorphism on $N$ to $M$ if for any finite nonempty subsets $S$ and $T$ of $Q, \pi(S) \leqq \Sigma(T)$ implies $\pi(f(S)) \leqq \Sigma(f(T))$. This follows immediately from the fact that by Lemma $1.9, \pi(S) \leqq \Sigma(T)$ only when $\alpha \leqq \beta$ for some $\alpha \in S, \beta \in T$.

THEOREM 1.12. Suppose $P=\bigcup_{k \in K} P_{k}$, where for each $k$, every element of $P_{k}$ is incomparable with every element of $P-P_{k}$. Then $P^{*}$ is the free product of the $P_{k}^{*}$.

Proof. By Theorem 1.11, we may assume $P_{k}^{*}$ is a sublattice of $P^{*}$. Let $\varphi_{k}: P_{k}^{*} \rightarrow P^{*}$ be the inclusion map, and let $f_{k}: P_{k}^{*} \rightarrow M$ be a homomorphism into a distributive lattice $M$. Let $g: P \rightarrow M$ be the union of the restrictions $f_{k} \mid P_{k}$. Then $g$ is order preserving, and can be extended to a homomorphism $h: P^{*} \rightarrow M$. We have $h \mid P_{h}^{*}=f_{k}$, and the proof is complete.

\section{Conditionally implicative lattices.}

Definition 2.1. If $x$ and $y$ are members of a lattice, $x \rightarrow y$ denotes the largest element $z$ such that $x z \leqq y$.

DEFINITION 2.2. A lattice $L$ is implicative if $x \rightarrow y$ exists for all $x, y$ in $L$.

It is known that every implicative lattice is distributive and has a largest element. Note also that $x \rightarrow y \geqq y$.

Definition 2.3. A lattice $L$ is conditionally implicative if whenever $x, y$ are in $L$ and $x \not y$, then $x \rightarrow y$ exists. 
A conditionally implicative lattice with a largest element is implicative. If $L$ is a lattice and $L^{\prime}$ is obtained from $L$ by adding a largest element, then $L$ is conditionally indicative if and only if $L^{\prime}$ is implicative. It follows that a conditionally implicative lattice is distributive.

LEMMA 2.4. Let $x, y$, and $z$ be members of a distributive lattice. If $x \rightarrow y$ and $x \rightarrow z$ exist, then $x \rightarrow y z$ exists and equals $(x \rightarrow y)(x \rightarrow z)$. If $x \rightarrow z$ and $y \rightarrow z$ exist, then $(x+y) \rightarrow z$ exists and equals $(x \rightarrow z)(y \rightarrow z)$.

Proof. The proof is immediate.

THEOREM 2.5. If $L_{\alpha}$ is conditionally implicative for each $\alpha \in P$, then $\sum_{\alpha \in P} L_{\alpha}$ is conditionally implicative.

Proof. By (1.1) and Lemma 2.4, we need only show that if $\alpha_{1}, \cdots, \alpha_{m}$ are distinct members of $P, \beta_{1}, \cdots, \beta_{n}$ are distinct members of $P, i_{r} \in L_{\alpha_{r}}$ for $1 \leqq r \leqq m, j_{s} \in L_{\beta_{s}}$ for $1 \leqq s \leqq n, x=\varphi_{\alpha_{1}}\left(i_{1}\right) \cdots \cdot \varphi_{\alpha_{m}}\left(i_{m}\right)$, $y=\varphi_{\beta_{1}}\left(j_{1}\right)+\cdots+\varphi_{\beta_{n}}\left(j_{n}\right)$, and $x \not y$, then $x \rightarrow y$ exists. Indeed every member of $L$ is a sum of elements of type $x$ as well as a product of elements of type $y$. We may suppose $\alpha_{k}=\beta_{k}$ for $1 \leqq k \leqq p$ and $\left\{\alpha_{p+1}, \cdots, \alpha_{m}\right\}$ is disjoint from $\left\{\beta_{p+1}, \cdots, \beta_{n}\right\}$. Since $x \not y, i_{k} \not j_{k}$ for all $k$. Let

$$
z=\sum_{k=1}^{p} \varphi_{\alpha_{k}}\left(i_{k} \rightarrow j_{k}\right)+\sum_{k=p+1}^{n} \varphi_{\beta_{k}}\left(j_{k}\right) \text {. }
$$

Clearly $x z \leqq y$ and $y \leqq z$. Suppose $w=\varphi_{\gamma_{1}}\left(t_{1}\right) \cdot \cdots \cdot \varphi_{\gamma_{q}}\left(t_{q}\right)$ and $x w \leqq y$. By Lemma 1.9, either $w \leqq y$, hence $w \leqq z$, or for some $r, s, \gamma_{r}=\alpha_{s}=\beta_{s}$ and $i_{s} t_{r} \leqq j_{s}$. In the second case, $w \leqq \varphi_{r_{r}}\left(t_{r}\right) \leqq \varphi_{\alpha_{s}}\left(i_{s} \rightarrow j_{s}\right) \leqq z$. Since every element of $\Sigma_{\alpha \in P} L_{\alpha}$ is a sum of elements of type $w$, it follows that $x \rightarrow y=z$.

REMARK. It is easily seen using Lemma 1.9 that the converse of Theorem 2.5 also holds. Thus Theorem 2.10 below follows directly from Theorem 2.5 and its converse.

COROLlary 2.6. A free distributive lattice is conditionally implicative.

Proof. A free distributive lattice is the free product of one-element lattices.

Definition 2.7. A lattice $M$ is a retract of a lattice $L$ if there exist homomorphisms $f: L \rightarrow M$ and $g: M \rightarrow L$ such that $f g=I_{M}$.

THEOREM 2.8. If for some $\beta \in P, L_{\beta}$ has a largest and smallest element, then $L_{\beta}$ is a retract of $L=\sum_{\alpha \in P} L_{\alpha}$. If $P$ is trivially ordered, the same holds with no hypothesis on $L_{\beta}$. 
Proof. Let 0 and 1 be the smallest and largest elements of $L_{\beta}$. For each $\alpha$, define $f_{\alpha}: L_{\alpha} \rightarrow L_{\beta}$ by : for each $x \in L_{\alpha}$,

$$
\begin{array}{lll}
f_{\alpha}(x)=1 & \text { if } & \alpha>\beta \\
f_{\alpha}(x)=x & \text { if } & \alpha=\beta \\
f_{\alpha}(x)=0 & \text { if } & \alpha \nsupseteq \beta .
\end{array}
$$

The family $\left\{f_{\alpha} \mid \alpha \in P\right\}$ satisfies the hypothesis of (1.3). Therefore there exists a homomorphism $f: L \rightarrow L_{\beta}$ such that $f \varphi_{\beta}=f_{\beta}=I_{L_{\beta}}$. In case $P$ is trivially ordered, fix an element $x_{0}$ of $L_{\beta}$ and define $f_{\alpha}: L_{\alpha} \rightarrow L_{\beta}$ by

$$
\begin{array}{rll}
f_{\alpha}(x)=x_{0} & \text { if } & \alpha \neq \beta \\
=x & \text { if } & \alpha=\beta
\end{array}
$$

The rest of the proof is as before.

THEOREM 2.9. A retract $M$ of a conditionally implicative lattice $L$ is conditionally implicative.

Proof. Let $f$ and $g$ be homomorphisms as in Definition 2.7. Let $x, y$ be elements of $M$ with $x \notin y$. Then $g(x) \notin g(y)$, for otherwise $x=f g(x) \leqq f g(y)=y$. Let $z=f(g(x) \rightarrow g(y))$. We have $g(x)(g(x) \rightarrow$ $g(y)) \leqq g(y)$. Applying $f$ to both sides, we obtain $x z \leqq y$. Now suppose $x w \leqq y$. Then $g(x) g(w) \leqq g(y)$, and hence $g(w) \leqq g(x) \rightarrow g(y)$. Applying $f$, we find $w \leqq z$. Thus $z=x \rightarrow y$.

THEOREM 2.10. Let $L$ be the free product of a family $\left\{L_{\alpha} \mid \alpha \in I\right\}$ of distributive lattices. Then $L$ is conditionally implicative if and only if $L_{\alpha}$ is conditionally implicative for all $\alpha$.

Proof. This follows from Theorems 2.5, 2.8 and 2.9.

3. Order sums and projective lattices. In contrast to the situation for Boolean algebras, the direct product of two projective distributive lattices is not always projective, [1, Example 8.3]. As a replacement for this theorem, the results of this section show that projectivity is often preserved under order sums.

DEFINITION 3.1. A distributive lattice $L$ is projective if for every pair of distributive lattices $L_{1}$ and $L_{2}$, every homomorphism $h: L \rightarrow L_{2}$, and every epimorphism $f: L_{1} \rightarrow L_{2}$, there exists a homomorphism $g: L \rightarrow L_{1}$ such that $f g=h$.

The following lemmas are known and easily proved. 
LEMMA 3.2. A retract of a projective distributive lattice is projective.

LEMMA 3.3. If $L$ is projective and $f: L_{1} \rightarrow L$ is an epimorphism, then there exists a homomorphism $g: L \rightarrow L_{1}$ such that $f g=I_{L} . \quad$ In particular, every projective distributive lattice is a retract of a free distributive lattice.

THEOREM 3.4. The free product $L$ of a family $\left\{L_{i} \mid i \in I\right\}$ of distributive lattices is projective if and only if $L_{i}$ is projective for each $i \in I$.

Proof. The condition is necessary by Theorem 2.8 and Lemma 3.2. If the condition holds, let $M$ and $N$ be distributive lattices, $f: M \rightarrow N$ an epimorphism and $h: L \rightarrow N$ a homomorphism. For each $i \in I$, let $\varphi_{i}$ be the monomorphism of Definition 1.1. Since $L_{i}$ is projective, there exists a homomorphism $g_{i}: L_{i} \rightarrow M$ such that $f g_{i}=h \varphi_{i}$. There exists a homomorphism $g: L \rightarrow M$ such that $g \varphi_{i}=g_{i}$. Now for each $i, f g \varphi_{i}=f g_{i}=h \varphi_{i}$. By (1.1), it follows that $f g=h$.

Theorem 3.4 is a generalization of the following known statement.

LEMmA 3.5. A free distributive lattice is projective.

Proof. Since a one-element lattice is projective, the result follows from Theorem 3.4.

THEOREM 3.6. A projective distributive lattice is conditionally implicative.

Proof. This follows from Corollary 2.6, Lemma 3.3 and Theorem] 2.9 .

THeOREM 3.7. Suppose for each $\alpha \in P, M_{\alpha}$ is a retract of $L_{\alpha}$. Then $M=\sum_{\alpha \in P} M_{\alpha}$ is a retract of $L=\sum_{\alpha \in P} L_{\alpha}$.

Proof. By hypothesis there exist homomorphisms $f_{\alpha}: L_{\alpha} \rightarrow M_{\alpha}$ and $g_{\alpha}: M_{\alpha} \rightarrow L_{\alpha}$ such that $f_{\alpha} g_{\alpha}=I_{M \alpha_{\alpha}}$. Let $\phi_{\alpha}: L_{\alpha} \rightarrow L$ and $\psi_{\alpha}: M_{\alpha} \rightarrow M$ be the monomorphisms of Definition 1. Let $h_{\alpha}=\psi_{\alpha} f_{\alpha}$. By (1.3) there exists a homomorphism $f: L \rightarrow M$ such that $f \varphi_{\alpha}=h_{\alpha}, \alpha \in P$. Similarly there exists a homomorphism $g: M \rightarrow L$ such that $g \psi_{\alpha}=\varphi_{\alpha} g_{\alpha}$. For each $\alpha$, we have $f g \psi_{\alpha}=f \varphi_{\alpha} g_{\alpha}=\psi_{\alpha} f_{\alpha} g_{\alpha}=\psi_{\alpha}$. Since $M$ is generated by $\bigcup_{\alpha \in P} \psi_{\alpha}\left(M_{\alpha}\right)$, it follows that $f g=I_{M}$.

Lemma 3.8. If $P$ is a partially ordered set, then $P^{*}$ is projective if and only if for each $\alpha \in P$ there exists a finite sequence 
$S_{\alpha, 0}, \cdots, S_{\alpha, p(\alpha)}$ of nonempty finite subsets of $P$ such that

(3.1) $\alpha \in S_{\alpha, p(\alpha)}$, and every member of $S_{\alpha, p(\alpha)}$ is $\geqq \alpha$.

(3.2) for each $r, S_{\alpha, r}$ has a member $\leqq \alpha$.

(3.3) if $\alpha<\beta$, then for every $r$ there is an $s$ such that $S_{\alpha, r} \supseteqq$ $S_{\beta, s}$.

Proof. By [1, Th. 5.1], $P^{*}$ is projective if and only if for each $\alpha$ there exist nonempty finite subsets $S_{\alpha, 0}, \cdots, S_{\alpha, p(\alpha)}$ of $P$ such that (3.3) holds and

$$
\alpha=\sum_{r=0}^{p(\alpha)} \pi\left(S_{\alpha, r}\right)
$$

where the products and sum are relative to $P^{*}$. By Lemma 1.9, (3.4) is equivalent to (3.2) and

$$
\text { for some } r \text {, every element of } S_{\alpha, r} \text { is } \geqq \alpha \text {. }
$$

By renumbering the indices, we obtain (3.1) from (3.2) and (3.5).

TheOREM 3.9. Let $\left\{L_{\alpha} \mid \alpha \in P\right\}$ be a family of distributive lattices each of which have a smallest and largest element. Then $L=$ $\sum_{\alpha \in P} L_{\alpha}$ is projective if and only if $L_{\alpha}$ is projective for each $\alpha$, and $P^{*}$ is projective.

Proof. Suppose $L$ is projective. By Lemma 3.2 and Theorem 2.8, $L_{\alpha}$ is projective for each $\alpha$. Since a one-element lattice is a retract of any lattice, the projectivity of $P^{*}$ follows from Lemma 3.2 and Theorem 3.7.

For the converse, let $N$ be a free distributive lattice which is freely generated by a family $\left\{d_{\alpha, i} \mid \alpha \in P, i \in L_{\alpha}\right\}$ of distinct elements. Let $N_{\alpha}$ be the sublattice generated by $\left\{d_{\alpha, i} \mid i \in L_{\alpha}\right\}$, let $f_{\alpha}: N_{\alpha} \rightarrow \varphi_{\alpha}\left(L_{\alpha}\right)$ be the homomorphism such that $f_{\alpha}\left(d_{\alpha, i}\right)=\varphi_{\alpha}(i), i \in L_{\alpha}$, and let $f: N \rightarrow L$ be the extension of the $f_{\alpha}$. By Corollary 3.5 and Lemma 3.3, there exist homomorphisms $g_{\alpha}: \varphi_{\alpha}\left(L_{\alpha}\right) \rightarrow N_{\alpha}$ such that $f_{\alpha} g_{\alpha}=I_{\varphi\left(L_{\alpha}\right)}$. By Lemma 3.2, we need only find a homomorphism $g: L \rightarrow N$ such that $f g=I_{L}$.

For each $\alpha$, there exist sets $S_{\alpha, 1}, \cdots, S_{\alpha, p(\alpha)}$ as in Lemma 3.8 . For $i \in L_{\alpha}$, let

$$
\begin{aligned}
b_{\alpha, i, \beta} & =\varphi_{\alpha}(i) \quad \text { if } \quad \beta=\alpha \\
& =\varphi_{\beta}\left(0_{\beta}\right) \text { if } \quad \beta>\alpha \\
& =\varphi_{\beta}\left(1_{\beta}\right) \text { if } \quad \beta \nsupseteq \alpha,
\end{aligned}
$$

where $0_{\beta}, 1_{\beta}$ are the smallest and largest elements of $L_{\beta}$. Let 


$$
c_{\alpha, i}=\sum_{r=1}^{p(\alpha)} \prod_{\beta \in S_{\alpha, r}} g_{\beta}\left(b_{\alpha, i, \beta}\right) .
$$

We wish to show that $\left\{c_{\alpha, i} \mid \alpha \in P, i \in L_{\alpha}\right\}$ satisfies all the inequalities of the set $E$ defined in the proof of Theorem 1.7. First, suppose $i_{1} \cdot \cdots \cdot i_{m} \leqq j_{1}+\cdots+j_{n}$, where the elements involved are in $L_{\alpha}$. For each $i \in L_{\alpha}$, we have $c_{\alpha, i}=g_{\alpha}\left(\varphi_{\alpha(i)}\right) P_{\alpha}+Q_{\alpha}$, where $P_{\alpha}$ and $Q_{\alpha}$ are independent of $i$. Therefore

$$
\begin{aligned}
c_{\alpha, i_{1}} \cdots c_{\alpha, i_{m}} & =g_{\alpha} \varphi_{\alpha}\left(i_{1}\right) \cdots \cdot g_{\alpha} \varphi_{\alpha}\left(i_{m}\right) P_{\alpha}+Q_{\alpha} \\
& \leqq\left[g_{\alpha} \varphi_{\alpha}\left(j_{1}\right)+\cdots+g_{\alpha} \varphi_{\alpha}\left(j_{n}\right)\right] P_{\alpha}+Q_{\alpha} \\
& =c_{\alpha, j_{1}}+\cdots+c_{\alpha, j_{n}} .
\end{aligned}
$$

Next, suppose $\alpha<\beta, i \in L_{\alpha}$, and $j \in L_{\beta}$. To show $c_{\alpha, i} \leqq c_{\beta, j}$, first observe that for any $\gamma \in P, b_{\alpha, i, \gamma} \leqq b_{\beta, j, \gamma}$ (consider the cases $\gamma>\beta, \gamma=\beta$, and $\gamma \nsupseteq \beta$ ). By (3.3) for each $r, 0 \leqq r \leqq p(\alpha), S_{\alpha, r} \supseteqq S_{\beta, s}$ for some $s$. Therefore for every $r$,

$$
\prod_{\gamma \in S_{\alpha, r}} g_{\gamma}\left(b_{\alpha, i, \gamma}\right) \leqq \prod_{\gamma \in S_{\beta, s}} g_{\gamma}\left(b_{\alpha, i, \gamma}\right) \leqq \prod_{\gamma \in S_{\beta} s} g_{\gamma}\left(b_{\beta, j, \gamma}\right) \leqq c_{\beta, j} .
$$

Hence $c_{\alpha, i} \leqq c_{\beta, j}$.

By Theorem 1.6, there exists a homomorphism $g: L \rightarrow N$ such that $g\left(\varphi_{\alpha(i)}\right)=c_{\alpha, i}$ for all $\alpha \in P, i \in L_{\alpha}$. Now, since $f$ extends each $f_{\beta}$, we have for $\alpha \in P, i \in L_{\alpha}$,

$$
f g\left(\varphi_{\alpha}(i)\right)=f\left(c_{\alpha, i}\right)=\sum_{r=1}^{p(\alpha)} \prod_{\beta \in S_{\alpha, r}} b_{\alpha, i, \beta} .
$$

By (3.1) and (3.2), it is easily seen that the right side of (3.6) reduces to $\varphi_{\alpha}(i)$. Using (1.1), we conclude that $f g=I_{M}$.

We have been unable to find workable necessary and sufficient conditions for the projectivity of $P^{*}$. It is necessary that $P$ contain no uncountable chain, since no projective lattice can contain such a chain [1, Th. 4.2]. On the other hand, the countability of $P$ is a sufficient condition, as shown by the following.

THEOREM 3.10. If $P$ is countable, and $L_{\alpha}$ is a countable projective distributive lattice for each $\alpha \in P$, then $L=\sum_{\alpha \in P} L_{\alpha}$ is projective.

Proof. Let $N, N_{\alpha}, f_{\alpha}, f$, and $g_{\alpha}$ be as in the proof of Theorem 3.9. Arrange the members of $\bigcup_{\alpha \in P} \varphi_{\alpha}\left(L_{\alpha}\right)$ in a sequence $a_{1}, a_{2}, \cdots$ without repetitions. For each $n$, let $\alpha_{n}$ be the $\alpha$ such that $a_{n} \in \varphi_{\alpha}\left(L_{\alpha}\right)$. We define inductively:

$$
c_{1}=g_{\alpha_{1}}\left(a_{1}\right) \text {, }
$$


and for $n>1$,

$$
c_{n}=g_{\alpha_{n}}\left(a_{n}\right) \Pi\left\{c_{r} \mid r<n, \alpha_{r}>a_{n}\right\}+\sum\left\{c_{r} \mid r<n, \alpha_{r}<\alpha_{n}\right\} .
$$

We wish to show there exists a homomorphism $g: L \rightarrow N$ such that $g\left(a_{n}\right)=c_{n}$ for all $n$. By Theorem 1.6, this will be the case if we show:

If $\alpha_{m}<\alpha_{n}$, then $c_{m} \leqq c_{n}$, and

If $\alpha_{m_{1}}=\cdots=\alpha_{m_{p}}=\alpha_{n_{1}}=\cdots=\alpha_{n_{q}}$, and

$a_{m_{1}} \cdot \cdots \cdot a_{m_{p}} \leqq a_{n_{1}}+\cdots+a_{n_{q}}$, then

$c_{m_{1}} \cdot \cdots \cdot c_{m_{p}} \leqq c_{n_{1}}+\cdots+c_{n_{q}}$.

We prove (3.7) by proving the following by induction on $n$ :

If $m<n$, then $\alpha_{m}<\alpha_{n}$ implies $c_{m} \leqq c_{n}$, and $\alpha_{m}>\alpha_{n}$ implies $c_{m} \geqq c_{n}$.

For $n=1$, this holds vacuously. Suppose (3.9) holds for all $n<p$, and $p>1$. If $m<p$ and $\alpha_{m}<\alpha_{p}$, then $c_{m} \leqq \sum\left\{c_{r} \mid r<p, \alpha_{r}>\alpha_{p}\right\} \leqq c_{p}$, where the second inequality holds by definition of $c_{p}$. Suppose $m<p$ and $\alpha_{m}>\alpha_{p}$. Then $c_{m}$ is one of the factors of $g_{\alpha_{p}}\left(a_{p}\right) \Pi\left\{c_{r} \mid r<p, \alpha_{r}>\alpha_{p}\right\}$. Hence to show $c_{m} \geqq c_{p}$, we need only show $c_{m} \geqq c_{r}$ whenever $r<p$ and $\alpha_{r}<\alpha_{p}$. Since $\alpha_{m}>\alpha_{r}$ and $m<p, r<p$, this follows from the induction hypothesis.

As for (3.8), let $\alpha$ be the common value of $\alpha_{m_{1}}, \cdots, \alpha_{n_{q}}$. Replace each of $c_{m_{1}}, \cdots, c_{m_{p}}$ by its defining formula and distribute the product of these as a sum of products. We obtain $c_{m_{1}} \cdots \cdot c_{m_{p}}=A+B$, where

$$
A=g_{\alpha}\left(a_{m_{1}}\right) \Pi\left\{c_{r} \mid r<i_{1}, \alpha_{r}>\alpha\right\} \cdot \cdots g_{\alpha}\left(a_{m_{p}}\right) \Pi\left\{c_{r}\left|r<i_{p}\right| \alpha_{r}>\alpha\right\}
$$

and $B$ is a sum of products each of which has a factor $c_{r}$ with $\alpha_{r}<\alpha$. By (3.7), $B \leqq c_{n_{1}}$. It remains to show $A \leqq c_{n_{1}}+\cdots+c_{n_{q}}$. Using (3.7), we have

$$
c_{m_{1}} \cdot \cdots \cdot c_{m_{p}} \leqq \Pi\left\{c_{r} \mid r<j_{k}, \alpha_{k}>\alpha\right\} \text { for } 1 \leqq k \leqq q .
$$

Therefore

$$
\begin{aligned}
c_{n_{1}}+\cdots+c_{n_{q}} & \geqq \sum_{k=1}^{q}\left[g_{\alpha}\left(a_{n_{k}}\right) \Pi\left\{c_{r} \mid r>j_{k}, \alpha_{k}<\alpha\right\}\right] \\
& \geqq c_{m_{1}} \cdots \cdot c_{m_{p}} \sum_{k=1}^{q} g_{\alpha}\left(a_{n_{k}}\right) \\
& \geqq c_{m_{1}} \cdots \cdot c_{m_{p}} \prod_{k=1}^{p} g_{\alpha}\left(a_{m_{k}}\right) \\
& \geqq c_{m_{1}} \cdots \cdot c_{m_{p}} \cdot A=A .
\end{aligned}
$$


This proves the existence of the homomorphism $g$. By Lemma 3.2, we have only to prove $f g=I_{L}$. This will hold if we show

$$
f g\left(a_{n}\right)=a_{n}, \quad \text { for all } n .
$$

We have $f g\left(a_{1}\right)=f\left(c_{1}\right)=f g_{\alpha_{1}}\left(a_{1}\right)=f_{\alpha_{1}} g_{\alpha_{1}}\left(a_{1}\right)=a_{1}$. Suppose (3.10) holds for $n<p$, and $p>1$. Then

$$
\begin{aligned}
f g\left(a_{p}\right)= & f g_{\alpha_{p}}\left(a_{p}\right) \Pi\left\{f g\left(a_{r}\right) \mid r<p, \alpha_{r}>\alpha_{p}\right\} \\
& +\sum\left\{f g\left(a_{r}\right) \mid r<p, \alpha_{r}<\alpha_{p}\right\},
\end{aligned}
$$

which by the induction hypothesis,

$$
\begin{aligned}
& =a_{p} \Pi\left\{a_{r} \mid r<p, \alpha_{r}>\alpha_{p}\right\}+\sum\left\{a_{r} \mid r<p, \alpha_{r}<\alpha_{p}\right\} \\
& =a_{p},
\end{aligned}
$$

by (1.2).

CoROllary 3.11. If $P$ is countable, then $P^{*}$ is projective.

The next theorem gives another sufficient condition for the projectivity of $P^{*}$.

THEOREM 3.12. Let $P$ be partially ordered set such that:

$$
\begin{aligned}
& \text { for each } \alpha,\{\beta \mid \beta \geqq \alpha\} \text { is well ordered by } \leqq \text {, and } \\
& \text { if } \alpha<\beta \text {, then }\{\gamma \mid \alpha \leqq \gamma \leqq \beta\} \text { is finite. }
\end{aligned}
$$

Proof. For each $\alpha$, let $f(\alpha)=\alpha$ if $\alpha$ is a maximal member of $P$, and $f(\alpha)=$ the immediate successor of $\alpha$ otherwise. Let $f^{0}=I_{P}$, and for an integer $p>1, f^{P}=f f^{p-1}$. Let us write $\alpha \sim \beta$ whenever for some $\gamma, \gamma \geqq \alpha$ and $\gamma \geqq \beta$. By (3.11), $\sim$ is an equivalence relation. Let $\left\{P_{k} \mid k \in K\right\}$ be the set of all $\sim$ equivalence classes. By Theorem $1.12, P^{*}$ is the free product of $\left\{P_{k}^{*} \mid k \in K\right\}$. Therefore by Theorem 3.4, we may assume $\alpha \sim \beta$ for all $\alpha, \beta$ in $P$.

Fix an element $\alpha_{0}$ of $P$. For each $\alpha$, let $p(\alpha)$ be the smallest integer $n \geqq 0$ such that $f^{n}\left(\alpha_{0}\right) \geqq \alpha$, and let $q(\alpha)$ be the smallest integer $n \geqq 0$ such that $f^{n}(\alpha)=f^{p(\alpha)}\left(\alpha_{0}\right)$. For $0 \leqq r \leqq p(\alpha)$, let

$$
S_{\alpha, r}=\left\{f^{r}\left(\alpha_{0}\right)\right\} \cup\left\{f^{n}\left(\alpha_{0}\right) \mid 0 \leqq n \leqq q(\alpha)-1\right\} \text {. }
$$

Conditions (3.1) and (3.2) of Lemma 3.8 clearly hold. To verify (3.3), we need only show that for each $r$ there is an $s$ such that $S_{\alpha, r} \supseteqq$ $s_{f(\alpha), s}$. If $\alpha_{0} \not \leq \alpha$, then $p(f(\alpha))=p(\alpha)$ and $q(f(\alpha))=q(\alpha)-1$, and for each $r, S_{\alpha, r} \supseteqq S_{f(\alpha), s}$. If $\alpha_{0} \leqq \alpha$, then $\alpha=f^{p(\alpha)}\left(\alpha_{0}\right), p(f(\alpha))=$ 
$p(\alpha)+1, q(f(\alpha))=q(\alpha)=0$, and $S_{\alpha, r}=S_{f(\alpha), r}$ for each $r \leqq p(\alpha)$. Thus by Lemma $3.8, P^{*}$ is projective.

We return to the question of the projectivity of order sums of projective lattices.

THEOREM 3.13. If $L_{0}$ and $L_{1}$ are free distributive lattices, $L_{0}$ is uncountable, and $L_{1}$ is infinite, then the ordinal sum $L_{0} \oplus L$, is not projective.

Proof. We may assume $L_{0}$ and $L_{1}$ are disjoint. Let $L_{0}$ and $L_{1}$ be freely generated by $A=\left\{\alpha_{\alpha} \mid \alpha \in I\right\}$ and $B=\left\{b_{\beta} \mid \beta \in J\right\}$ respectively. If $L_{0} \oplus L_{1}$ is projective, then by [1, Corollary 5.2, and remark following Th. 5.1] for each $\alpha$ and $\beta$ there exist finite sequences $S_{\alpha, 0}, \cdots, S_{\alpha, p(\alpha)}$ and $S_{\beta, 0}, \cdots, S_{\beta, p}(\beta)$ of non-empty finite subsets of $A \cup B$ such that:

$$
\begin{aligned}
a_{\alpha} & =\sum_{r=0}^{p(\alpha)} \Pi\left(S_{\alpha, r}\right) \\
b_{\beta} & =\sum_{r=0}^{p(\beta)} \Pi\left(S_{\beta, r}\right) \\
\text { if } \quad r & \neq s, S_{\beta, r} \nsubseteq S_{\beta, s}
\end{aligned}
$$

for each $\alpha, \beta$ and each $r, 0 \leqq r \leqq p(\alpha)$, there exists an $s, 0 \leqq s \leqq p(\beta)$, such that $S_{\alpha, r} \supseteqq S_{\beta, s}$.

Since $a_{\alpha}<b_{\beta}$ for all $\alpha, \beta$ and $B$ is independent, it follows from (3.14) that $S_{\beta, r}=\left\{b_{\beta}\right\}$ for some $r$, say $r=0$. If $0<r \leqq p(\beta)$, $\Pi\left(S_{\beta, r}\right) \leqq b_{\beta}$ and $b_{\beta} \notin S_{\beta, r}$ by (3.15). Therefore by the independence of $B, S_{\beta, r} \cap A \neq \varnothing$ for $r>0$.

Let $\beta_{1}, \beta_{2}, \cdots$ be a sequence of distinct members of $J$. For each integer $n>0$, let

$$
\Gamma_{n}=\bigcup_{r=1}^{p\left(\beta_{n}\right)} S_{\beta_{n}, r} \cap A
$$

Since $\Gamma_{n}$ is countable, there is an $\alpha \in A$ such that $a_{\alpha} \notin \Gamma_{n}$. If we show

$$
b_{\beta_{n}} \in \bigcup_{r=0}^{p(\alpha)} S_{\alpha, r}
$$

for all $n$, we will have a contradiction since this union is finite.

Suppose (3.17) fails for some $n$. By (3.16) for $0 \leqq r \leqq p(\alpha)$, either $S_{\alpha, r} \supseteqq S_{\beta_{n, 0}}=\left\{b_{\beta_{n}}\right\}$, or $S_{\alpha, r} \supseteqq S_{\beta_{n}, s}$ for some $s>0$. The first case is impossible by assumption. Hence for each $r, S_{\alpha, r}$ contains an element $a_{\alpha}$ of $S_{\beta_{n}, s} \cap A$. By (3.13), $a_{\alpha} \leqq \sum_{r=0}^{p(\alpha)} a_{\alpha_{r}}$. Since $A$ is inde- 
pendent, we have $\alpha=\alpha_{r}$ for some $r$ and therefore $a_{\alpha} \in \Gamma_{n}$, which is a contradiction.

Definition 3.14. If $L$ is a lattice, let $D(L)$ be the dual of $L$, that is, $L$ with reverse order.

The dual of a projective (free) distributive lattice is projective (free).

In contrast to Theorem 3.13, we have the following.

THEOREM 3.15. If $L_{0}$ and $L_{1}$ are projective and either $L_{0}$ has a largest element or $L_{1}$ has a smallest element, then $L=L_{0} \oplus L_{1}$ is projective.

Proof. We may assume $L_{0}$ and $L_{1}$ are disjoint. Let $N$ be a free distributive lattice with distinct free generators $\left\{d_{0, i} \mid i \in L_{0}\right\} \cup\left\{d_{1, i} \mid i \in L_{1}\right\}$. For $r=0,1$, let $N_{r}$ be the sublattice generated by $\left\{d_{r, i} \mid i \in L_{r}\right\}$, and let $f_{r}: N_{r} \rightarrow L_{r}$ be the homomorphism such that $f_{r}\left(d_{r, i}\right)=i$. Let $f: N \rightarrow L$ be the homomorphism which extends $f_{0}$ and $f_{1}$. By Lemma 3.3, there exists homomorphisms $g_{r}=L_{r} \rightarrow N_{r}$ such that $f_{r} g_{r}=I_{L_{r}}$.

Suppose $L_{0}$ has a largest element 1. Define $g: L \rightarrow N$ by

$$
\begin{aligned}
g(x) & =g_{0}(x), \quad \text { if } x \in L_{0} \\
& =g_{0}(1)+g_{1}(x), \quad \text { if } x \in L_{1} .
\end{aligned}
$$

It is easily seen that $g$ is a homomorphism and $f g=I_{L}$. Hence $L$ is projective. If $L_{1}$ has a smallest element, then $D\left(L_{1}\right) \oplus D\left(L_{0}\right)$ is projective, since $D\left(L_{1}\right)$ has a largest element. Therefore $L=$ $D\left(D\left(L_{1}\right) \oplus D\left(L_{0}\right)\right)$ is projective.

Corollary 3.16. Let $L_{0}$ and $L_{1}$ be free distributive lattices. Then $L=L_{0} \oplus L_{1}$ is projective if and only if either

(i ) $L_{0}$ and $L_{1}$ are countable, or

(ii) $L_{0}$ or $L_{1}$ is finite.

Proof. If (i) holds, $L$ is projective by Theorem 3.10. If (ii) holds, $L$ is projective by Theorem 3.15. Suppose neither (i) nor (ii) holds. Then one of $L_{0}, L_{1}$ is uncountable and the other is infinite. In this case $L$ is not projective by Theorem 3.13 and duality.

COROLlary 3.17. Let $L_{0}$ and $L_{1}$ be uncountable free distributive lattices, and $\{a\}$ be a one-element lattice. Then $L_{0} \oplus L_{1}$ is not projective, but $L_{0} \oplus\{a\} \oplus L_{1}$ is projective.

Proof. This follows from Theorems 3.13 and 3.15 and the associativity of ordinal sums. 


\section{REFERENCES}

1. R. Balbes, Projective and injective distributive lattices, Pacific J. Math. 21 (1967), 405-420.

2. R. Sikorski, Products of abstract algebras, Fund. Math. 39 (1952), 221-218.

Received August 22, 1966. Part of this material appears in the first author's doctoral dissertation. The research was supported in part by National Science Foundation Grants Nos. 4038 and 5600.

UNIVERSITY OF CALIForNia, LOS ANGELES 



\section{PACIFIC JOURNAL OF MATHEMATICS}

\section{EDITORS}

\section{H. SAMELSON}

Stanford University

Stanford, California

\author{
J. P. JANS \\ University of Washington \\ Seattle, Washington 98105
}

\section{J. DuGUNDJI}

University of Southern California

Los Angeles, California 90007

RichaRd ARENS

University of California

Los Angeles, California 90024

\section{ASSOCIATE EDITORS}

E. F. BECKENBACH
B. H. NeUManN

F. WOLF

K. YOSIDA

\section{SUPPORTING INSTITUTIONS}

UNIVERSITY OF BRITISH COLUMBIA

CALIFORNIA INSTITUTE OF TECHNOLOGY

UNIVERSITY OF CALIFORNIA

MONTANA STATE UNIVERSITY

UNIVERSITY OF NEVADA

NEW MEXICO STATE UNIVERSITY

OREGON STATE UNIVERSITY

UNIVERSITY OF OREGON

OSAKA UNIVERSITY

UNIVERSITY OF SOUTHERN CALIFORNIA
STANFORD UNIVERSITY

UNIVERSITY OF TOKYO

UNIVERSITY OF UTAH

WASHINGTON STATE UNIVERSITY

UNIVERSITY OF WASHINGTON

AMERICAN MATHEMATICAL SOCIETY

CHEVRON RESEARCH CORPORATION

TRW SYSTEMS

NAVAL ORDNANCE TEST STATION

Mathematical papers intended for publication in the Pacific Journal of Mathematics should be typewritten (double spaced). The first paragraph or two must be capable of being used separately as a synopsis of the entire paper. It should not contain references to the bibliography. Manuscripts may be sent to any one of the four editors. All other communications to the editors should be addressed to the managing editor, Richard Arens at the University of California, Los Angeles, California 90024.

50 reprints per author of each article are furnished free of charge; additional copies may be obtained at cost in multiples of 50 .

The Pacific Journal of Mathematics is published monthly. Effective with Volume 16 the price per volume ( 3 numbers) is $\$ 8.00$; single issues, $\$ 3.00$. Special price for current issues to individual faculty members of supporting institutions and to individual members of the American Mathematical Society: $\$ 4.00$ per volume; single issues $\$ 1.50$. Back numbers are available.

Subscriptions, orders for back numbers, and changes of address should be sent to Pacific Journal of Mathematics, 103 Highland Boulevard, Berkeley 8, California.

Printed at Kokusai Bunken Insatsusha (International Academic Printing Co., Ltd.), No. 6, 2-chome, Fujimi-cho, Chiyoda-ku, Tokyo, Japan.

PUBLISHED BY PACIFIC JOURNAL OF MATHEMATICS, A NON-PROFIT CORPORATION

The Supporting Institutions listed above contribute to the cost of publication of this Journal, but they are not owners or publishers and have no responsibility for its content or policies. 


\section{Pacific Journal of Mathematics}

\section{Vol. 21, No. $3 \quad$ BadMonth, 1967}

Richard Allen Askey, A transplantation theorem for Jacobi coefficients . . . 393

Raymond Balbes, Projective and injective distributive lattices .......... 405

Raymond Balbes and Alfred Horn, Order sums of distributive lattices . . . . 421

Donald Charles Benson, Nonconstant locally recurrent functions ........ 437

Allen Richard Bernstein, Invariant subspaces of polynomially compact operators on Banach space ............................... 445

Robert F. Brown, Fixed points and fibre ................... 465

David Geoffrey Cantor, On the Stone-Weierstrass approximation theorem for valued fields ................................ 473

James Walton England, Stability in topological dynamics .............. 479

Alessandro Figà-Talamanca and Daniel Rider, A theorem on random

Fourier series on noncommutative groups.................. 487

Sav Roman Harasymiv, A note of dilations in $L^{p} \ldots \ldots \ldots \ldots \ldots \ldots . \ldots 493$

J. G. Kalbfleisch, A uniqueness theorem for edge-chromatic graphs ....... 503

Richard Paul Kelisky and Theodore Joseph Rivlin, Iterates of Bernstein polynomials .................................... 511

D. G. Larman, On the union of two starshaped sets ............... 521

Henry B. Mann, Josephine Mitchell and Lowell Schoenfeld, Properties of differential forms in $n$ real variables ...................... 525

John W. Moon and Leo Moser, Generating oriented graphs by means of team comparisons .

Veikko Nevanlinna, A refinement of Selberg's asymptotic equation ...

Ulrich Oberst, Relative satellites and derived functors of functors with additive domain ..............................

John Vincent Ryff, On Muirhead's theorem...............

Carroll O. Wilde and Klaus G. Witz, Invariant means and the Stone-Čech compactification 